\title{
PLAN DIRECTOR PARA LA REGIÓN DE SIERRAS CHICAS, CÓRDOBA, ARGENTINA
}

\section{MASTER PLAN FOR THE REGION OF SIERRAS CHICAS, CÓRDOBA, ARGENTINA}

IRÓS, Guillermo M.

Instituto de Planificación del Área Metropolitana - Gobierno de la Provincia de Córdoba

Arquitecto, Coordinador General

Rufino Cuervo 1085, Oficina 15, C.P. 5010, Córdoba Capital, Argentina

Correo electrónico: iplamprovincia@gmail.com

Teléfono: +54 0351-4347791

MOISO, Enrique A.

Instituto de Planificación del Área Metropolitana - Gobierno de la Provincia de Córdoba Arquitecto, Director de Gestión Institucional

ALONSO, César S.

Instituto de Planificación del Área Metropolitana - Gobierno de la Provincia de Córdoba Arquitecto, Director de Proyectos Estratégicos

\section{BRAVO, Augusto 0.}

Instituto de Planificación del Área Metropolitana - Gobierno de la Provincia de Córdoba Arquitecto, Director de Planificación

Palabras Clave: Planificación; Planificación Regional; Planificación Metropolitana; Plan Director

Key words: Planning; Regional Planning; Metropolitan Planning; Master Plan

\section{Resumen}

El Plan Director de Sierras Chicas fue una experiencia innovadora en planificación regional, desarrollada por un Consejo de Planificación Metropolitana, coordinado por el Gobierno de la Provincia de Córdoba e integrado, además de los organismos técnicos provinciales, por los gobiernos municipales involucrados en el área de estudio; universidades; organizaciones sociales y profesionales. Con un horizonte temporal de planificación aproximado de 15 años, el Plan aplica sobre un sector particular del Área Metropolitana de Córdoba conocido como Sierras Chicas, una conurbación integrada por 11 Municipalidades y Comunas recostadas sobre el faldeo oriental de las sierras, en el cuadrante noroeste metropolitano. Se trata de un corredor que ha experimentado un crecimiento poblacional explosivo y desordenado en las últimas décadas, con consecuencias que afectan sus sobresalientes condiciones ambientales, paisajísticas y culturales, patrimonio que deben preservarse para beneficio de los habitantes y visitantes, actuales y futuros. Su alcance regional implica la integración en un espacio geográfico amplio de distintas competencias territoriales municipales. La planificación enfocada en la escala regional permite abordar una multiplicidad de aspectos que hacen a un desarrollo armónico de las ciudades. Cuencas, excedentes hídricos, agua potable, cloacas, energía, transporte, ordenación territorial, vialidad, equipamientos sociales y muchas otras variables que podrían ampliar esta lista, resultan imposibles de ser estudiadas y resultas dentro de los recortes de cada radio municipal. 


\section{Abstract}

The Master Plan for Sierras Chicas was an innovative experience in regional planning. It was developed by a Metropolitan Planning Council, Provincial technical entities, governments of the cities included in the area under analysis, universities, professional and social entities, all of them coordinated by the Government of the Province of Cordoba. With an approximate time, span of 15 years, the Plan applies to a specific sector of the Metropolitan Area of Cordoba known as Sierras Chicas. This conurbation is made up of 11 cities and communes that lie on the eastern slope of the hills in the northwest quadrant of the metropolitan area. This corridor has undergone a disorderly and explosive population growth in the past decades as a consequence affecting its outstanding cultural, landscape, and environmental conditions, which are a heritage that should be preserved for future and current visitors and inhabitants. It has regional impact on the integration in a vast geographical space with different city territorial competencies. If planning is focused on the region, a number of aspects can be approached for a harmonic development of cities. It's impossible to analyze and plan efficiently for basins, surplus water, drinking water, sewage, energy, transport, territory ordering, roads, social equipment, and many other variables, if such analysis is made on each city in isolation.

\section{Introducción}

\subsection{Alcances y motivos}

Resulta importante en esta breve introducción, destacar el valor de un Plan Director regional que comprende los distintos espacios geográficos que delimitan las competencias territoriales municipales. La planificación enfocada en la escala regional permite abordar una multiplicidad de aspectos que hacen a un desarrollo armónico de las ciudades. Cuencas, excedentes hídricos, agua potable, cloacas, energía, transporte, ordenación territorial, vialidad, equipamientos sociales y muchas otras variables que podrían ampliar esta lista, resultan imposibles de ser estudiadas y resultas dentro de los recortes de cada radio municipal.

En otro sentido la planificación regional promueve una lectura amplia que abarca las diferentes causas que en la mayoría de los casos convergen para la generación de fenómenos o procesos de orden tanto físico como social. La consideración conjunta de esa variedad de causas permite generar acciones que desde distintos ángulos colaboran en la solución de un problema. Se evitan también de esta forma, diagnósticos que resultan equivocados por haber considerado solo aspectos parciales en el análisis.

Si esto es importante en general para las áreas metropolitanas, resulta absolutamente imprescindible en situaciones de continuidad de los tejidos urbanos o de conurbación (usando el término acuñado por Patrick Geddes). ${ }^{1}$ En particular para las siete primeras ciudades del cuadrante noroeste de la ciudad de Córdoba que conforman el corredor de Sierras Chicas. Se destaca finalmente que, si bien se hizo mención a la necesidad de enfocar el análisis y las propuestas en el espacio regional, también es importante resaltar la necesidad de sumar la mirada local y la orientación que cada ciudad propone desde la escala urbana como complemento del Plan Director.

1 Geddes, Patrick. Ciudades en evolución. Buenos Aires: Infinito, 1960. 


\section{2 Área de estudio}

La subregión metropolitana conocida como Sierras Chicas es un continuo urbano que se desarrolla hacia el noroeste de la Ciudad de Córdoba, siguiendo básicamente dos corredores viales que a su vez acompañan el faldeo oriental de las sierras: la Ruta Provincial E57, de carácter "interno" a los asentamientos urbanos, antiguamente un ramal ferroviario; y la Ruta Provincial E53.

Figura 1. Sierras Chicas en el Área Metropolitana de Córdoba

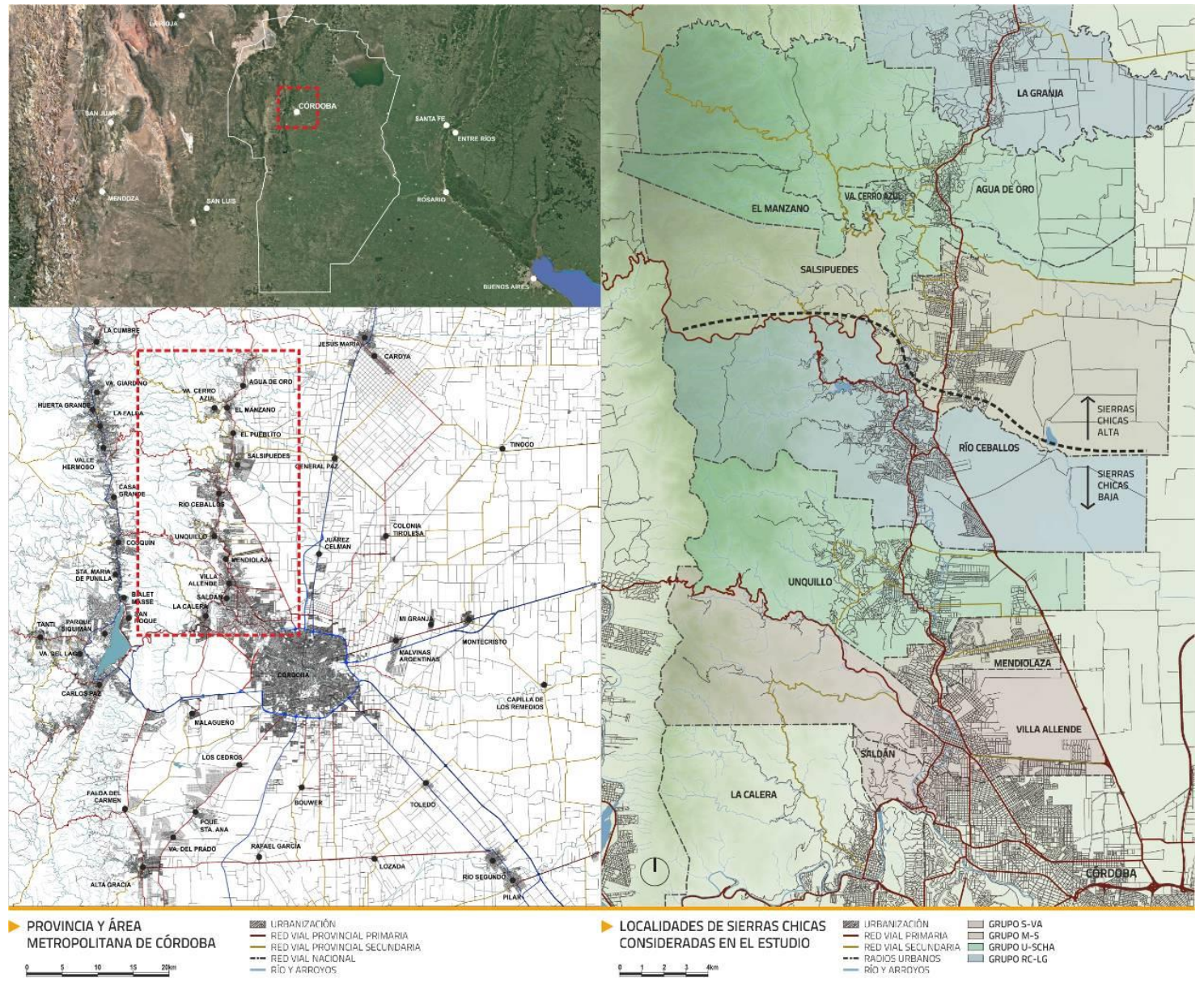

Fuente: Elaboración propia

Concretamente, al corredor lo conforman 11 localidades, además de la Ciudad de Córdoba. Ordenadas según la mayor proximidad a la cuidad madre, la secuencia se inicia con La Calera, Saldán y Villa Allende en contacto directo con el ejido capitalino, y luego siguiendo los corredores ya mencionados hacia el noroeste metropolitano se ubican Mendiolaza, Unquillo, Río Ceballos, Salsipueses, El Manzano, Agua de Oro, Cerro Azul y La Granja.

\subsection{Metodología}

Las tendencias verificadas, permitirán esbozar un diagnóstico y un pronóstico a futuro, no obstante, los lineamientos que aquí se expresan no deben considerarse un marco referencial estático e inmodificable. Hacerlo sería caer en el error de no advertir las inevitables

Citación: IRÓS, G. et al. Plan Director para la Región de Sierras Chicas, Córdoba, Argentina. En: Libro de proceedings, CTV 2018. XII Congreso Internacional Ciudad y Territorio Virtual. "Ciudades y Territorios Inteligentes". UNCuyo, Mendoza, 5-7 septiembre 2018. Barcelona: CPSV, 2018, p. 29-44. 
alteraciones que las circunstancias, muchas veces imponderables o imprevisibles, produce el devenir del tiempo.

Por esta razón queda planteado desde el inicio la necesidad de realizar correcciones y adecuaciones periódicas para acompañar el contexto real que irá marcando el proceso de transformación.

Para no ingresar en situaciones de indefinición y desorden, atento que este Plan Director contará con el correspondiente marco legal, se establecen periodos de cinco años para iniciar el proceso de revisión y actualización. A su vez al cumplimentarse una revisión se prorroga por igual tiempo el plazo final de 15 años que expresa el horizonte de planificación. De esta forma el Plan Director de Sierras Chicas adopta el carácter de permanente.

Como mecánica operativa se establece un procedimiento que contempla la participación coordinada del gobierno provincial a través de sus distintos organismos según competencias específicas; los gobiernos municipales; universidades; organizaciones sociales y profesionales. A tales efectos se constituyó el COPLAMET (Consejo de Planificación Metropolitana) con la participación de los sectores mencionados, según las modalidades que aconsejen los temas a tratar.

Desde este enfoque, el Plan Sierras Chicas asume el carácter de Plan Director y como tal, define las Políticas de Estado para un desarrollo sustentable de esta sub región. El mismo contiene los Planes Particulares y complementariamente, desde las municipalidades, o los organismos provinciales específicos o en forma conjunta según corresponda, oportunamente se elaborarán los Programas y Proyectos correspondientes como emergentes de los mismos.

Esta metodología plantea como premisa la necesidad de alcanzar consensos amplios a partir del respeto a las autonomías municipalidades. Esto demanda acuerdos inter jurisdiccionales que garanticen la incorporación de las distintas particularidades locales en el marco regional, haciendo viable y efectiva la gestión de un Plan Director.

Otro tanto sucede con la consideración de los aportes valiosos que surgen de las Organizaciones Sociales; Profesionales y las Universidades.

Los Planes Particulares que finalmente integran el Plan Director son los siguientes:

- Patrimonio Ambiental

- Desarrollo Sociocultural Y Productivo

- Movilidad Y Conectividad

- Recursos Hídricos, Agua Potable, Efluentes Y Escorrentías

- Energía Eléctrica, Gas Y Recursos No Convencionales

- Residuos Sólidos Urbanos

- Gestión De Riesgo Y Seguridad Ciudadana

- Ordenamiento Urbano Y Territorial

Citación: IRÓs, G. et al. Plan Director para la Región de Sierras Chicas, Córdoba, Argentina. En: Libro de proceedings, CTV 2018. XII Congreso Internacional Ciudad y Territorio Virtual. "Ciudades y Territorios Inteligentes". UNCuyo, Mendoza, 5-7 septiembre 2018. Barcelona: CPSV, 2018, p. 29-44. 


\section{Diagnóstico general}

A continuación, se describen sintéticamente los principales problemas territoriales detectados por los distintos Planes Particulares que integran el documento final.

\subsection{Fuerte impacto de la urbanización sobre el soporte ambiental}

Sierras Chicas se asienta sobre un soporte natural de características singulares dentro del Área Metropolitana de Córdoba. Las condiciones hidrológicas, geomorfológicas y fitogeográficas han influido en los asentamientos humanos desde las culturas aborígenes hasta la actualidad.

Las suaves ondulaciones del piedemonte oriental de las Sierras Chicas, así como los valles inter serranos, constituyeron un escenario apreciado por la feracidad de sus valles, la madera de los bosques, los frutos silvestres, la miel, el agua de los numerosos arroyos y las favorables condiciones para el cultivo del maíz.

La conformación urbano-territorial actual mantiene un significativo correlato con la condición natural, extendiéndose en forma lineal sobre el faldeo de la sierra y los cauces de ríos y arroyos. El conflicto paradójico reside en que el crecimiento urbano generado por la atracción de los atributos paisajísticos es la principal amenaza para los mismos.

Las consecuencias que pueden sobrevenir por un crecimiento no planificado, puede llevar a la pérdida de muchas de aquellas cualidades que motivaron la elección de estas ciudades de Sierras Chicas. Se trata del consumo indiscriminado del recurso natural que reduce un capital que se expresa en los bosques, el agua, el aire y la biodiversidad entre otras variables.

A continuación, se detallan los principales procesos conflictivos identificados dentro de esta complejidad ambiental, todos interrelacionados y concatenados tal como ocurre en un sistema ambiental complejo.

- Deterioro de la vegetación nativa: Aquel magnífico Espinal que albergó ejemplares arbóreos de gran porte acompañado por un denso arbustal, ha sido arrasado por la explotación agrícola y la urbanización. En la gran Ilanura productiva de la Provincia solo perduran algunos relictos en forma de archipiélagos o fragmentos aislados, pero el bosque serrano y los pastizales de altura no han sido modificados significativamente por la actividad agrícola. Allí, los procesos de consumo de bosque se relacionan con la deforestación para uso de leña, la minería, los incendios, el sobre-pastoreo y de manera significativa la urbanización. El mayor consumo de suelo por crecimiento urbano en Sierras Chicas se produjo sobre áreas con cobertura de bosque nativo. Sólo en los últimos años, inducidos por los costos económicos y sociales relacionados con la urbanización de las sierras, se ha comenzado a extender la mancha urbana sobre la llanura Este, consolidando viejos loteos y habilitando nuevas expansiones.

- Pérdida de capacidad absorbente del suelo: Directamente asociado a la pérdida de cobertura vegetal nativa se presenta la pérdida de la capacidad del suelo de infiltración del agua de lluvia. En los factores que modifican y deterioran la cobertura vegetal del suelo se encontrarán seguramente muchas de las explicaciones a consecuencias ambientales negativas como los aumentos de escorrentías superficiales y caudales de crecidas de ríos y 
arroyos entre otras. Particularmente en Sierras Chicas, este fenómeno negativo es potenciado por las elevadas pendientes que presenta la topografía. Si bien no son cerros relativamente altos (la diferencia entre los extremos y el fondo de la cuenca no es mayor a $500 \mathrm{~m}$ ), sí presentan laderas abruptas que aumentan la velocidad de escurrimiento y dificultan los procesos naturales de infiltración.

- Crecidas y sequías de arroyos: La ya de por si escasa oferta del recurso hídrico, se ve amenazada y profundizada por esta modificación de las capas superficiales del suelo, que ha perdido gran parte de la cubierta herbácea, arbustal y boscosa. Las rápidas crecidas de los ríos y arroyos llevan como contracara una pronta disminución de sus caudales y hasta su desaparición en temporadas de sequía al haberse reducido la capacidad de recarga de las cuencas. Las crecientes repentinas producidas ante la ocurrencia de lluvias intensas se explican por las pendientes pronunciadas y la baja permeabilidad. En períodos de precipitaciones extraordinarias se producen episodios de inundación con torrentes destructivos que llaman a revisar los límites de urbanización en las distintas ciudades conurbadas.

- Crisis hídrica en el consumo de agua: El sistema de abastecimiento de agua para consumo en Sierras Chica se encuentra desde hace años seriamente comprometido. Por empezar, la región ha perdido definitivamente la posibilidad de autoabastecerse, por lo que cualquier solución al problema actual implica traer el recurso de otro lado. Fuera de esa solución, solo resta ajustar las pautas de consumo, ya considerablemente reducidas en comparación con otras comunidades metropolitanas: mientras la Ciudad de Córdoba registra actualmente un consumo diario promedio de $350 \mathrm{lts} / \mathrm{hab}$, con picos de 500 en verano, Sierras Chicas ha logrado registros por debajo de los $200 \mathrm{Its} / \mathrm{hab}$, un dato alentador, pero todavía lejos de los $100 \mathrm{lts} /$ hab que establece como suficiente la OMS.

\subsection{Crecimiento disperso de población con desequilibrios territoriales}

A mediados del siglo XX, la transformación industrial que experimenta la Ciudad de Córdoba le induce un crecimiento poblacional explosivo que la aleja indefectiblemente del resto de las localidades de la región. Comienza a conformarse un área metropolitana marcadamente macro cefálica.

En ese contexto, Sierras Chicas mantiene durante gran parte del siglo anterior una tasa de crecimiento moderada, empujada por su calidad paisajística siempre atractiva para la clase media-alta cordobesa y por la instalación de algunas industrias grandes, como las mineras y la misma Compañía Sud Americana de Cemento Portland en Dumesnil, que atraen una importante masa de población obrera.

A finales del siglo $X X$, la tendencia verificada durante muchos años que ubicaba a la ciudad Capital protagonizando los mayores porcentajes de incremento poblacional de la provincia, se ve marcadamente modificada. El cambio se explica por el éxodo desde Córdoba hacia las ciudades metropolitanas, en donde se destacan particularmente las de Sierras Chicas, aunque no de manera excluyente.

El proceso reconoce una variedad de causas entre las que se destacan, como históricamente ha ocurrido para esta región en estudio, aquellas vinculadas a las preferencias por los atributos del lugar.

Citación: IRÓS, G. et al. Plan Director para la Región de Sierras Chicas, Córdoba, Argentina. En: Libro de proceedings, CTV 2018. XII Congreso Internacional Ciudad y Territorio Virtual. "Ciudades y Territorios Inteligentes". UNCuyo, Mendoza, 5-7 septiembre 2018. Barcelona: CPSV, 2018, p. 29-44. 
Figura 2. Crecimiento urbano e impacto sobre el soporte natural

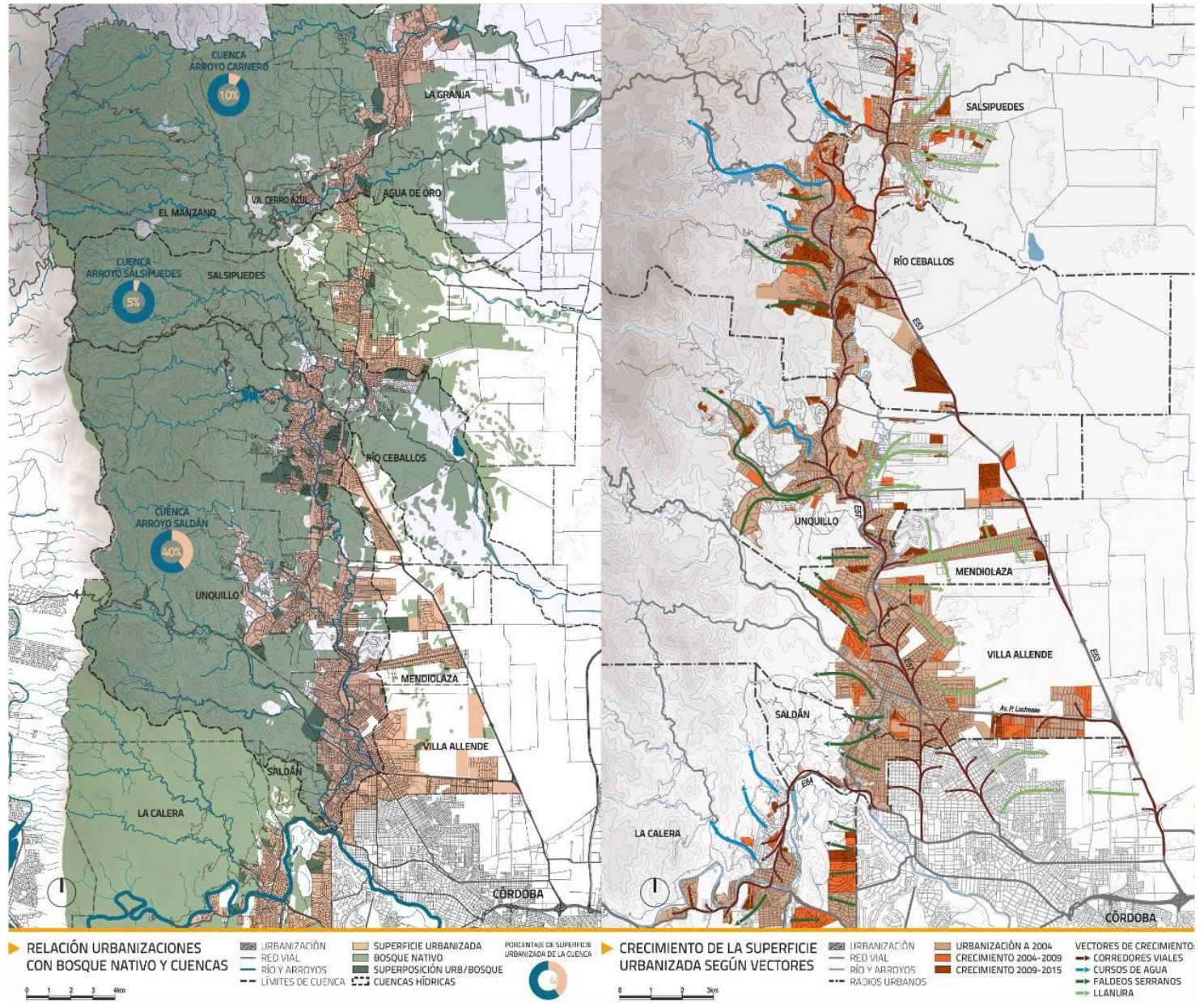

Fuente: Elaboración propia

Desde el censo del año 2010 hasta 2016 se han sumado cerca de 30.000 habitantes en Sierras Chicas. En el mismo intervalo de tiempo la ciudad de Córdoba incorpora aproximadamente 80.000; es decir 2,6 veces lo que sumó Sierras Chicas siendo que la ciudad Capital tiene a la fecha del censo 11 veces la población de este conjunto de ciudades.

Según cálculos del IPLAM, la relación entre la población proyectada al 2016 y la superficie urbanizada según imágenes satelitales de la misma época, arroja una densidad promedio de 20 hab/has para Sierras Chicas Sur y 9 hab/has para Sierras Chicas Norte. Valores muy bajos, aún comparados con los 60 hab/has de densidad promedio de la Ciudad de Córdoba, que confirman el proceso de dispersión de la urbanización y la habilitación de loteos impulsados por la dinámica de un mercado inmobiliario muy activo.

Si se parte del valor que asigna el censo de 2010 para las 11 localidades que involucra el Plan Director, proyectado al 2025, surge un incremento aproximado de 70.000 nuevos habitantes. Solo a efectos de simular un resultado, podemos asumir una hipótesis de 20 habitantes/hectárea como promedio de los distintos valores obtenidos para definir un área a urbanizar con esa densidad. Con esos valores, el incremento de superficie urbanizada desde 2010 a 2025 sería de 3.500 hectáreas, 35\% más de la superficie ocupada actualmente.

Citación: IRÓS, G. et al. Plan Director para la Región de Sierras Chicas, Córdoba, Argentina. En: Libro de proceedings, CTV 2018. XII Congreso Internacional Ciudad y Territorio Virtual. "Ciudades y Territorios Inteligentes". UNCuyo, Mendoza, 5-7 septiembre 2018. Barcelona: CPSV, 2018, p. 29-44. 
Como puede observarse, cualquiera de las magnitudes consignadas tanto en población como en superficie de urbanización, se aproxima o supera a las ciudades más grandes de la conurbación de Sierras Chicas. Un dato lo suficientemente elocuente que advierte sobre el impacto enorme que adquiere el proceso en un lapso relativamente breve.

\subsection{Deficiencias en la movilidad regional}

En las escalas actuales de desarrollo urbano, que no solo expande la ciudad hacia su periferia cercana, sino que la extiende hasta alcanzar a otras poblaciones anteriormente aisladas conformando sistemas de ciudades, el concepto de movilidad adquiere un sentido más amplio y complejo que debe ser complementado con el de conectividad.

La movilidad urbana y regional constituye uno de los aspectos que en la actualidad han adquirido una destacada significación en la planificación orientada al desarrollo sustentable. Cuando las escalas urbanas han superados las posibilidades de vinculación basadas en relaciones de proximidad, la disponibilidad de medios para desplazarse adquiere una dimensión distinta.

Esta situación se verifica en Sierras Chicas con una importancia mayor a la de otros corredores metropolitanos por la extensión territorial de la conurbación, el nivel de conflictividad actual del tránsito y el agravamiento que sobrevendrá, de no recurrirse a nuevas formas de movilidad, acompañadas de otros cambios urbanísticos y culturales².

El crecimiento se ha dado mayoritariamente en sectores residenciales de familias que mantienen sus lasos laborales y dependen de diferentes servicios que ofrece la ciudad de Córdoba, obligándolos a viajar con alta frecuencia; en muchos casos en forma cotidiana.

La red de caminos con algunas mejoras y modificaciones sigue siendo semejante a la que servía esos antiguos pueblos pequeños, y en el proceso de urbanización se fueron sumando fracciones sin una matriz de inter conexión prefigurada, lo que produce un resultado de extensiones urbanizadas conformadas por fragmentos inconexos.

La fuerte configuración radial metropolitana se reproduce en el corredor de $\mathrm{SCH}$, con una marcada ausencia de conexiones transversales entre las dos vías estructurantes del sector, la E57 conurbada en el fondo de cuenca, y la E53 por el llano del Este.

La estructura de la trama urbana también atenta con las facilidades de movilidad. A los quiebres de traza inducidos por la topografía y los cruces de arroyos y escorrentías se le suma el diseño urbano aislado y arbitrario.

El transporte público no ofrece condiciones de frecuencia, velocidad comercial y confort suficientes como para seducir al usuario para adoptarlo en reemplazo del automóvil. Ante esto, el automóvil particular registra un alto porcentaje de uso como medio de transporte habitual, protagonizando una mayor ocupación de la superficie vial disponible.

2 Irós, Guillermo [et al]. Hacia un transporte urbano - metropolitano integrado: Propuesta para el cuadrante de Sierras Chicas, Córdoba, Argentina. Córdoba: Ministerio de Infraestructura del Gobierno de la Provincia de Córdoba, 2014. 
La Encuesta de Origen y Destino del Área Metropolitana de Córdoba ${ }^{3}$, desarrollada en el 2009 por la Secretaría de Transporte de la Municipalidad de Córdoba y el PTUMA, muestra en el análisis de los datos sobre Modos de Transporte que para conexiones inter zonales, que vinculan a Córdoba con las otras localidades del Área Metropolitana, un $90 \%$ de desplazamientos se realizan en colectivos, autos y motos ( $45 \%$ colectivos, $40 \%$ autos y $5 \%$ motos). Pero al momento de considerar la importancia del auto en la ocupación de la vía, surge la real dimensión de la incidencia de ese $40 \%$ en la conflictividad del tránsito. Teniendo en cuenta la superficie de ocupación estimada según el medio utilizado, se detecta la prioridad absoluta del auto particular en comparación con los otros modos. Ocupando más del $75 \%$ de la vía, el automóvil transporta el $21 \%$ de los viajeros por hora; mientras que ocupando sólo el $7 \%$ del espacio viario, el ómnibus transporta a más del $70 \%$ de las personas en movimiento.

\section{Propuestas}

A continuación, se describen sintéticamente las principales propuestas desarrolladas por los distintos Planes Particulares que integran el documento final.

\subsection{Mitigación del impacto de la urbanización sobre el soporte físico- ambiental}

Este conflicto tiene dos aristas, por un lado, el soporte natural del corredor, deteriorado en su contacto con la mancha urbana, impermeabilizado, contaminado, debilitado ambientalmente y desprotegido ante el crecimiento no planificado de la ciudad; y por otro la urbanización que avanza sobre ese soporte, con patrones de ocupación dispersos y pautas de consumo de recursos insostenibles.

En este apartado se hará referencia a la primera mitad del conflicto, la relacionada con el soporte natural. Durante el desarrollo de la siguiente problemática, relacionada con el crecimiento disperso de la urbanización, se abordará la otra perspectiva, es decir, el comportamiento de la mancha urbana respecto al territorio que consume.

Las propuestas orientadas a minimizar el impacto de la urbanización sobre el soporte ambiental se estructuran a partir de dos grupos de proyectos. Por un lado, se propone recuperar y fortalecer la vegetación nativa, tanto en zona serrana y de piedemonte como dentro de la misma superficie urbanizada, en el fondo de la cuenca; y por otro lado se plantea la necesidad de regular el comportamiento del sistema hídrico, principalmente el control de las escorrentías superficiales durante las precipitaciones.

\subsubsection{Recuperación de la vegetación nativa}

La Ley Provincial 9.814 de Ordenamiento Territorial de Bosques Nativos, en sintonía con la Ley Nacional 26.331 de Presupuestos Mínimos de Protección Ambiental de los Bosques Nativos, consideran bosques nativos a los ecosistemas forestales naturales compuestos predominantemente por especies arbóreas nativas maduras, con diversas especies de flora y fauna asociadas, en conjunto con el medio que las rodea -suelo, subsuelo, atmósfera, clima, recursos hídricos-, conformando una trama interdependiente con características propias y

\footnotetext{
3 De Beláustegui, Humberto. Encuesta Origen Destino 2009: Movilidad en el Área Metropolitana de Córdoba. Buenos Aires: Proyecto de Transporte Urbano de Buenos Aires, 2011.
} 
múltiples funciones, que en su estado natural le otorgan al sistema una condición de equilibrio dinámico y que brinda diversos servicios ambientales a la sociedad.

Con ese criterio, definen tres categorías de conservación según la calidad detectada por telemetría. La Categoría I (rojo) aplica en sectores de muy alto valor de conservación, que no pueden desmontarse ni manejarse con fines productivos; la Categoría II (amarillo) aplica en sectores de mediano valor de conservación que no pueden desmontarse y que podrán ser sometidos a actividades de conservación, recuperación, recreativas, aprovechamiento de productos madereros y no madereros, sistemas silvo-pastoriles, recolección e investigación científica; y la Categoría III (verde) aplica en sectores de bajo valor de conservación que pueden transformarse parcialmente o en su totalidad. Esta última sería la única categoría que permite cambios en el uso del suelo y, por lo tanto, es factible de ser urbanizada.

Por medio del llamado Corredor Bio-geográfico de Sierras Chicas, el Plan prevé proteger la gran superficie nativa serrana de cuenca alta englobando las distintas parcelas que la componen en una única unidad ambiental. El primer desafío de este proyecto es el de definir los límites de dicho territorio, al tiempo de sostener en dicha delimitación el concepto de "corredor". Para tal efecto, se recurrió al concepto integrador de micro-cuenca.

Las tres principales cuencas que participan de la región de Sierras Chicas son las del Río Suquía, Río Carnero y Río Jesús María, pero todas lo hacen en parte, generalmente las subcuencas altas de las mismas. Todas trascienden al corredor, exceden los límites administrativos de las localidades que consideramos como parte de Sierras Chicas e inclusive se desarrollan sobre otros Departamentos provinciales.

Por esto anterior, es necesario apelar a divisiones más pequeñas para definir los límites del Corredor. Surge así el concepto de micro-cuenca, asociadas a cursos de agua menores que se van sumando para terminar alimentando los principales cuerpos de agua de la región. La microcuenca en sí misma es una unidad ambiental integral, ya que vincula e interrelaciona topografía, calidad de suelo, cubierta vegetal, escorrentía superficial y subterránea, fauna y actividades humanas (incluida la urbanización).

La propuesta se complementa con el proyecto de Parque Lineal Metropolitano, un espacio verde público que acompaña al curso del arroyo Saldán-Río Ceballos desde el Río Suquía hasta el Dique La Quebrada. En el Sur se conecta a la Reserva Natural de la Defensa "La Calera", al Norte alcanza la Reserva Hídrica Natural Parque La Quebrada creada por disposición provincial. Se constituye como un corredor ambiental que atraviesa los principales centros urbanos y a su vez vincula importantes áreas naturales protegidas pertenecientes al gran corredor biogeográfico desarrollado anteriormente.

En realidad, aborda ambos aspectos de la problemática ambiental tratada en este apartado: el conflicto sobre el soporte natural y el conflicto hidrológico. Por un lado, se constituye como un eje ambiental que integra los componentes naturales, principalmente agua y vegetación con los tejidos urbanos, las instituciones y los elementos de valor patrimonial, aumentando la disponibilidad de espacio público de calidad, como instrumento para facilitar las relaciones comunitarias y el desarrollo de variadas actividades para diferentes edades y preferencias. $Y$ por otro lado, libera áreas eventualmente inundables para amortiguación de crecidas y facilita su correcto acondicionamiento y mantenimiento, mejorando el comportamiento hidrológico general de cuerpo de agua.

Citación: IRÓS, G. et al. Plan Director para la Región de Sierras Chicas, Córdoba, Argentina. En: Libro de proceedings, CTV 2018. XII Congreso Internacional Ciudad y Territorio Virtual. "Ciudades y Territorios Inteligentes". UNCuyo, Mendoza, 5-7 septiembre 2018. Barcelona: CPSV, 2018, p. 29-44. 


\subsubsection{Regulación de escorrentías}

Las crecidas repentinas en la zona de Sierras Chicas constituyen la principal amenaza natural para este aglomerado urbano. En el Plan se aclara que estos fenómenos están condicionados por la baja permeabilidad del material geológico de las cuencas altas de dichas sierras, por la fuerte energía del relieve (grandes pendientes), la escasa vegetación natural y las condiciones climáticas del sector que generan precipitaciones localizadas e intensas por efectos orográficos. Estas características naturales de la región sumadas a la poca planificación y a la urbanización de zonas propensas a ser ocupadas por crecidas poco frecuentes llevan a que se produzcan catástrofes como las ocurridas en febrero de 2015.

El Plan define dos tipos básicos de medidas para lograr el manejo y control del drenaje pluvial: estructurales y no estructurales.

Las medidas estructurales se relacionan con la ejecución de obras hidráulicas, tanto en la cuenca hidrográfica como sobre los cursos de agua que actúan de colectores principales del sistema.

Una de estas obras consiste en la generación de Embalses y micro-embalses de regulación en la zona media y alta. Por la configuración geo mórfica del territorio, durante una precipitación, las micro-cuencas que componen el corredor de Sierras Chicas tienden a "cargar" los cauces rápidamente en la zona alta y media de su recorrido, ingresando a las zonas urbanas bajas ya con caudales muy elevados. Por esta razón, las principales medidas estructurales para mejorar el comportamiento hidrológico de la cuenda deben tomarse "río arriba", en las partes altas.

Otra obra estructural es el Saneamiento del cauce en la zona baja. Cuando los arroyos ingresan a la superficie urbanizada, localizada principalmente sobre el fondo de la cuenca, las posibilidades de retardo o retención son prácticamente nulas. La poca disponibilidad de espacio y el gran caudal que traen los cuerpos de agua sólo posibilitan un espectro muy reducido de medidas estructurales, básicamente orientadas a facilitar el transporte rápido del agua hasta la finalmente sacarla de la cuenca. Es por ello que, en estos sectores, las obras están asociadas a minimizar los obstáculos sobre el cauce, como puentes, vados y grandes árboles; y mejorar la capacidad de escurrimiento del canal.

Las medidas no estructurales son de tipo preventivo y presuponen una convivencia razonable de la población con los problemas derivados de los procesos naturales. Las mismas intentan compatibilizar los costos de obras a ejecutarse con los recursos realmente disponibles. Las medidas no estructurales típicas son la zonificación de áreas inundables, el pronóstico de crecidas, el monitoreo del sistema (tanto en lo referente a la cantidad como a la calidad del agua y el ambiente), el desarrollo de legislación tendiente a controlar del desarrollo urbano actual y futuro y la educación y concientización, entre otros.

\subsection{Desarrollo urbano con equilibrio territorial}

Las consecuencias del crecimiento poblacional remiten a las características cualitativas del desarrollo urbano que, en muchos aspectos, ha generado o agudizado un conjunto de problemas ambientales, falencias en las infraestructuras y la calidad de los servicios públicos. Situaciones estas, que en parte se explican por el crecimiento explosivo de población, pero también se relacionan con las formas o modos en que ese crecimiento se ve reflejado en la ocupación del territorio.

Citación: IRÓS, G. et al. Plan Director para la Región de Sierras Chicas, Córdoba, Argentina. En: Libro de proceedings, CTV 2018. XII Congreso Internacional Ciudad y Territorio Virtual. "Ciudades y Territorios Inteligentes". UNCuyo, Mendoza, 5-7 septiembre 2018. Barcelona: CPSV, 2018, p. 29-44. 
Se hace aquí referencia a la desproporcionada utilización de suelo en relación a la población que se aloja por la baja densidad que caracteriza en general a las nuevas urbanizaciones, al porcentaje importante de parcelas urbanizadas no construidas, la fragmentación, la discontinuidad y el desorden por falta de direccionamiento hacia modelos preestablecidos.

\subsubsection{Densificación y ocupación de terreno vacante}

El completamiento de parcelas vacantes en urbanizaciones existentes es sin ninguna duda la mejor alternativa para orientar el crecimiento hacia un modelo sustentable de ciudad compacta. Los instrumentos impositivos pueden alentar esa tendencia y desalentar la comercialización especulativa de la tierra. Tal vez no es factible plantear como meta la ocupación total de la actual mancha urbanizada, pero existe un punto razonable de equilibrio que tampoco está en otro extremo con una cantidad de suelo urbanizado ocioso que permitiría duplicar la población existente sin nuevos loteos.

Un proceso alternativo y a la vez complementario es el de "levantar" los parámetros normativos de tejido para generar mayor densidad de ocupación en áreas próximas o intersticiales a las ya urbanizadas. Inclusive, en Sierras Chicas ya se puede discutir sobre la generación de áreas de media densidad en vivienda colectiva. Esto constituye una alternativa para aumentar la densidad definiendo enclaves con infraestructura que no interfieran a los entornos vecinos y que a su vez permiten concentrar la superficie construida y liberar suelo para espacio verde.

\subsubsection{Control del crecimiento urbano}

La ocupación de áreas vacantes, la fijación de parámetros de ocupación más densos o la redensificación de áreas consolidadas no contienen la totalidad del crecimiento poblacional esperado. Indefectiblemente muchas de estas localidades seguirán creciendo en extensión, el desafío es conducir dicho crecimiento a través de estrategias conjuntas que establezcan parámetros compatibles entre aspectos ambientales y la ordenación del territorio.

En términos de límites, las localidades de Sierras Chicas se encuentran absolutamente conurbadas, por lo que los crecimientos hacia el norte o el sur ya han encontrado los límites municipales. Hacia el oeste, la Ley Provincial 9.814 de Ordenamiento Territorial de Bosques Nativos ha restringido severamente las posibilidades de crecimiento ocupando el faldeo oriental de las sierras. Sólo han quedado habilitados algunos emprendimientos con permisos ya emitidos sin evitar un importante repudio social por el hecho.

Esta situación es relativamente nueva en término legales, ya que históricamente sólo pequeñas porciones del territorio local se encontraban sujetas a jurisdicciones supra-municipales: los cursos de agua, algunas rutas, el ferrocarril y algún predio especial. El impacto de la restricción es aún mayor debido a que con el proceso de redefinición de los radios municipales iniciado en 2008, los gobiernos locales contaron súbitamente con grandes territorios sobre las cuales podían legislar y disponer si deseaban ocupar. A partir de la Ley de Bosque, el Estado Provincial tiene atribuciones sobre las definiciones de uso de amplias superficies municipales, y esas definiciones apuntan a una fuerte restricción a la ocupación urbana.

Frente a este panorama, el desafío en la conducción del crecimiento urbano en Sierras Chicas recae sobre la llanura del este, ocupada en forma desestructurada por urbanizaciones de distintos tipos y tramas conviviendo con islas de bosque nativo y una cada vez menos desarrollada actividad productiva. 
Figura 3. Propuesta de Corredor Biogeográfico, Parque Lineal Metropolitano y sistema de lagunas de retardo sobre la cuenca (Izquierda). Propuesta Usos del Suelo (Derecha)

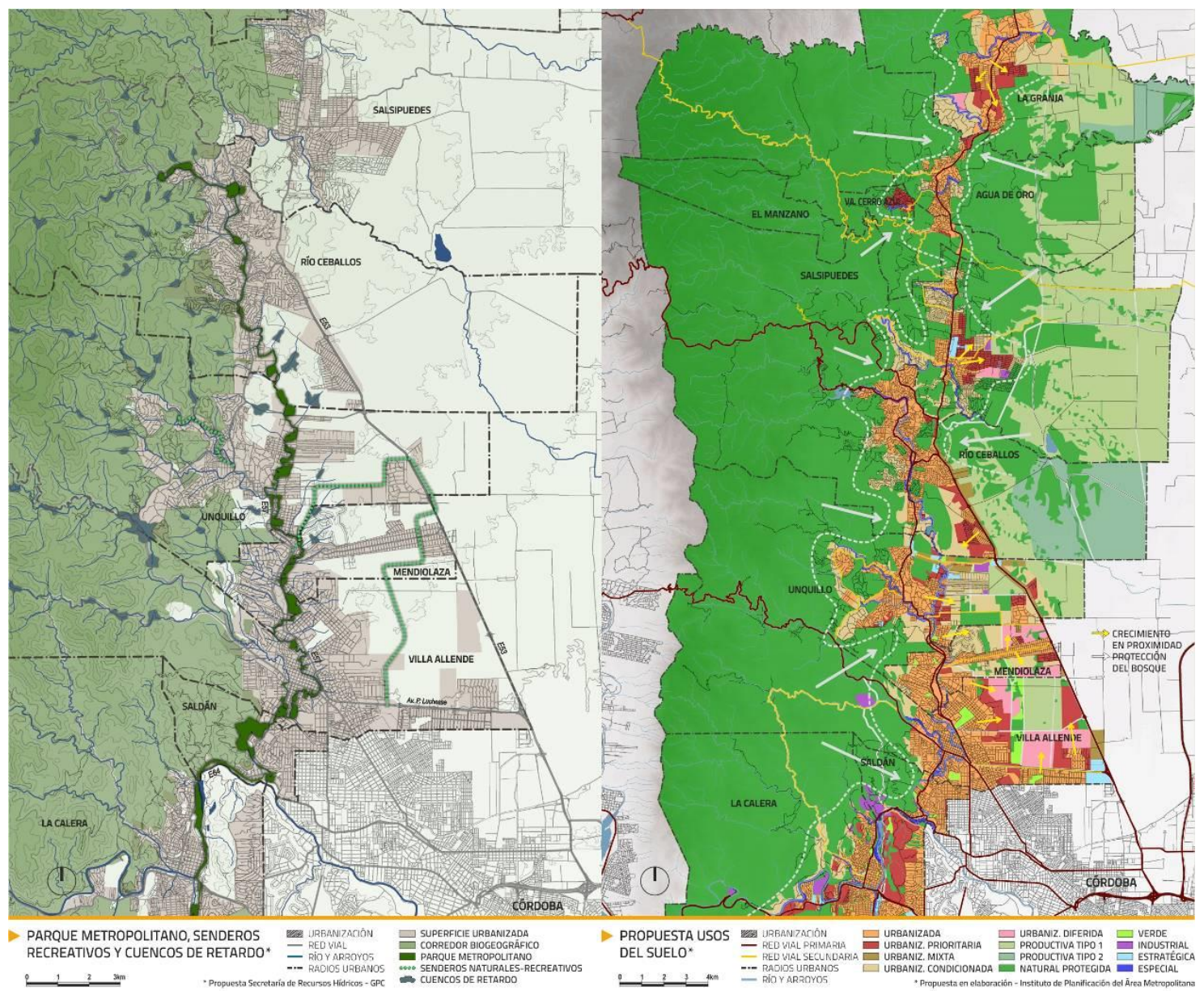

Fuente: Elaboración propia

Ante esta situación, el Plan Director propone un mapa de usos del suelo consensuado con los organismos públicos municipales y provinciales, discutido con organizaciones sociales y enriquecido con el aporte de profesionales e instituciones académicas. Se intentó alcanzar un balance medido entre la definición de un límite definitivo para el borde occidental de la urbanización contra el corredor Biogeográfico y la ocupación y reestructuración de la llanura oriental como la natural y futura expansión de la mancha urbana.

\subsection{Movilidad regional eficiente}

La movilidad urbana y regional constituye uno de los aspectos que en la actualidad han adquirido una destacada significación en la planificación orientada al desarrollo sustentable. Cuando las escalas urbanas han superados las posibilidades de vinculación basadas en relaciones de proximidad, la disponibilidad de medios para desplazarse adquiere una dimensión distinta.

Esta situación se verifica en Sierras Chicas con una importancia mayor a la de otros corredores metropolitanos por la extensión territorial de la conurbación, el nivel de conflictividad actual del tránsito y el agravamiento que sobrevendrá, de no recurrirse a nuevas formas de movilidad, acompañadas de otros cambios urbanísticos y culturales.

Citación: IRÓS, G. et al. Plan Director para la Región de Sierras Chicas, Córdoba, Argentina. En: Libro de proceedings, CTV 2018. XII Congreso Internacional Ciudad y Territorio Virtual. "Ciudades y Territorios Inteligentes". UNCuyo, Mendoza, 5-7 septiembre 2018. Barcelona: CPSV, 2018, p. 29-44. 


\subsubsection{Nueva red inter-conectiva}

El corredor se encuentra estructurado actualmente por dos vías regionales importantes que circulan con sentido Norte-Sur. La ruta E57, que sigue el fondo de la cuenca y sobre la que se desarrolla en continuo urbano de las localidades de Sierras Chicas Sur; y la ruta E53, que configurada como autovía de alta velocidad atraviesa la llanura del Este para luego estructurar las localidades de Sierras Chicas Norte. Ambos corredores, junto con la ruta E64 que forma el límite Sur del área de estudio, participan, y padecen, del esquema fuertemente radial que caracteriza el sistema viario del Área Metropolitana de Córdoba. Forman parte de los "rayos" que penetran a la ciudad capital desde los $360^{\circ}$ de su entorno regional, en este caso conectando el cuadrante noroeste. Estos tres corredores concentran todos los flujos: no hay otra forma de circular desde o hacia las localidades de Sierras Chicas si no es optando por alguno de ellos.

Las propuestas de conectividad expresadas en el Plan de Sierras Chidas están orientadas a reconstruir una retícula regional, generando conectores en ambos sentidos, N-S y E-O, que colaboren con los ya constituidos para terminar de armar una red o macro trama que multiplique las alternativas de conexión y evite la concentración de flujos en pocas arterias. Este sistema a su vez debe continuar vinculando la sub-región con el resto del Área Metropolitana, a través de conexiones con las sierras hacia el Oeste y conexiones con la llanura hacia el Este.

\subsubsection{Sistema integrado de transporte público regional}

Sierras Chicas cuenta con un único sistema de transporte público, el autobús. La mayoría de las localidades cuentan con dos modalidades de servicios de colectivos, urbanos, con una 0 más líneas que recorren internamente cada localidad; e interurbanos, con buses que vinculan el corredor con la Ciudad de Córdoba. Ambos sistemas, tal como ocurre en la misma ciudad Capital, se encuentran disociados, funcionan independientemente, no comparten paradas, tarifas ni recorridos.

Al hablar de un sistema integrado, se indica la necesidad de ensamblar a las distintas escalas y modalidades del transporte. En este caso, el esfuerzo consiste en combinar la escala urbana o local con la regional. Para ello, el Plan propone una serie de corredores troncales regionales de alta capacidad y eficiencia de servicio, alimentados por otros corredores menores que conectan los sectores barriales más alejados. El contacto entre ambos sistemas se produce a través de paradas y estaciones de transferencias, con coordinación de horarios y sistema único de pago.

Si bien el IPLAM ha considerado como matriz principal para un Sistema Metropolitano de Transporte Público Integrado al espacio ferroviario disponible en toda la región, Sierras Chicas merece un estudio particular. El cuadrante Noroeste que nos ocupa particularmente en este trabajo, queda contenido por el ramal A1, conocido como Tren de las Sierras y el Belgrano hacia el Norte conectando, en la escala metropolitana, a la ciudad de Córdoba con La Calera en el primer caso y con Jesús María en el segundo. El problema reside en que entre los ramales mencionados se encuentra un área urbana y suburbana de gran extensión sin disponibilidad de espacios ferroviarios.

Al no disponerse de corredores ferroviarios, para la implementación de nuevos servicios masivos de transporte público, se hace necesario considerar otras alternativas basadas en un rediseño de los ejes principales del sistema vial y su utilización parcial para la circulación diferenciada del transporte público, favoreciendo su velocidad comercial. Se propone entonces

Citación: IRÓS, G. et al. Plan Director para la Región de Sierras Chicas, Córdoba, Argentina. En: Libro de proceedings, CTV 2018. XII Congreso Internacional Ciudad y Territorio Virtual. "Ciudades y Territorios Inteligentes". UNCuyo, Mendoza, 5-7 septiembre 2018. Barcelona: CPSV, 2018, p. 29-44. 
complementar una tecnología de LRT (Light Rail Transit) o Tren Ligero sobre el espacio ferroviario, con una tecnología de BRT (Bus Rapid Transit) o autobús expreso sobre vías regionales. Ambas modalidades, trenes ligeros y buses expresos, funcionan como servicios troncales hacia distintos puntos de la región, alimentados por corredores urbanos locales e integrados, todos, en paradas y estaciones de transferencias.

Figura 5. Propuesta sistema vial (Izquierda). Propuesta de transporte público (Derecha)

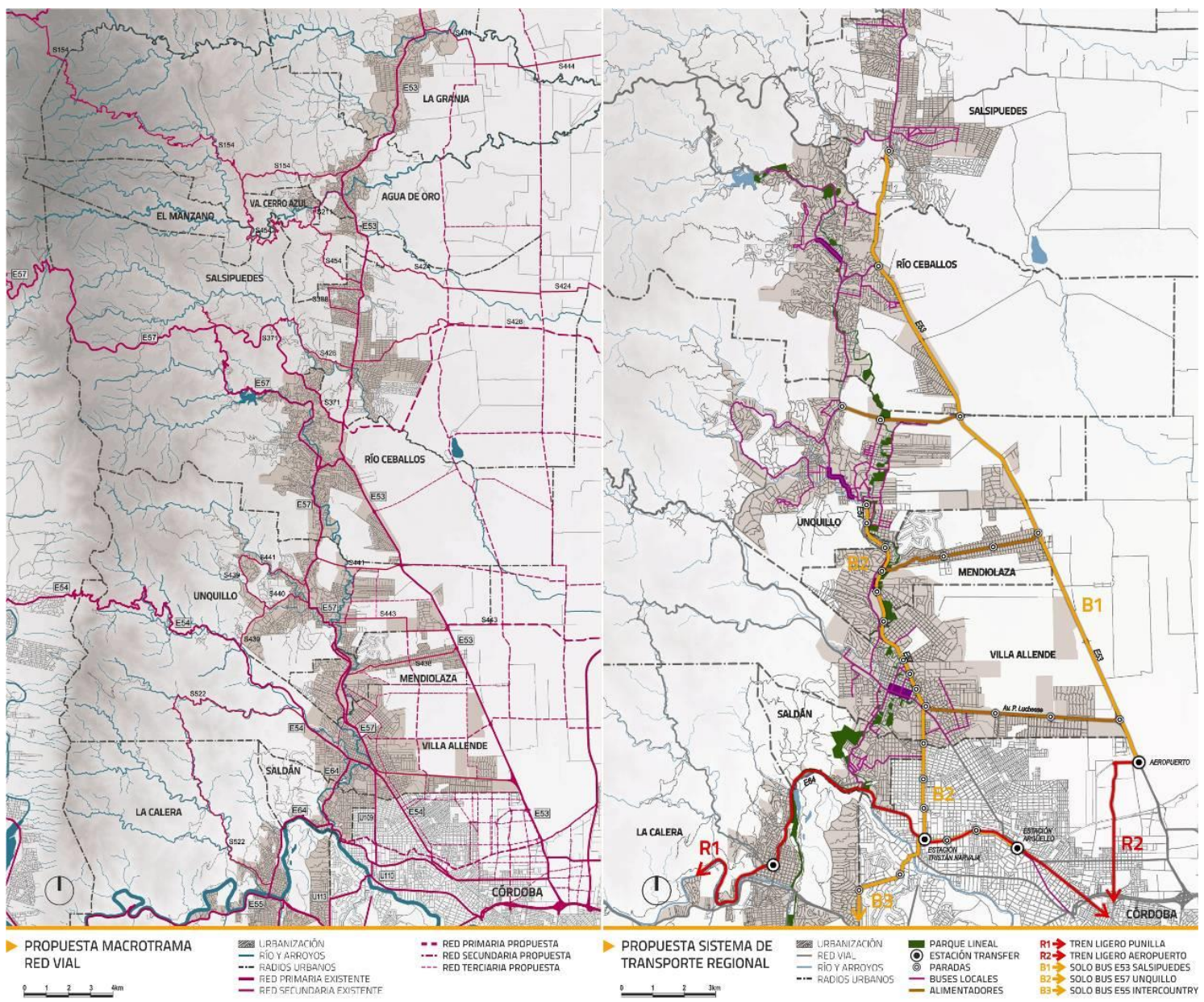

Fuente: Elaboración propia

\section{Conclusiones}

Este trabajo reúne los aportes de un numeroso conjunto de profesionales, especialistas, académicos de la UNC., técnicos y funcionarios de los distintos organismos del Estado Provincial, Municipalidades y Comunas de Sierras Chicas y numerosas organizaciones sociales y ciudadanos solidarios y participativos que han sumado su cooperación en el Consejo de Planificación Metropolitana, según las modalidades que requirieron los temas a tratados.

El resultado obtenido en este Plan Director de Sierras Chicas, reúne los lineamientos generales a seguir como Políticas de Estado, expresados en las directrices desarrolladas en los Planes Particulares. Queda así definida una orientación clara hacia una superación cualitativa de la Región desde una mirada que reúne la escala territorial y las particularidades de cada

Citación: IRÓS, G. et al. Plan Director para la Región de Sierras Chicas, Córdoba, Argentina. En: Libro de proceedings, CTV 2018. XII Congreso Internacional Ciudad y Territorio Virtual. "Ciudades y Territorios Inteligentes". UNCuyo, Mendoza, 5-7 septiembre 2018. Barcelona: CPSV, 2018, p. 29-44. 
localidad. Los avances y las circunstancias que lo acompañen, indicarán las correcciones o adecuaciones necesarias.

A partir de este Plan Director que define los lineamientos generales de planificación, se desarrollarán los proyectos y programas identificados, que encaucen el proceso en una instancia operativa y para alcanzar los objetivos. Los Programas y Proyectos no forman parte del presente Plan Director, y en consecuencia se abre ahora una nueva etapa; un nuevo proceso que será conducido por los organismos competentes y las municipalidades, recurriendo cuando la complejidad o especificidad lo requieran, al aporte de los institutos universitarios que cuentan con un calificado elenco de profesionales.

También se inicia una nueva etapa para el accionar del Consejo de Planificación Metropolitana que, en consonancia con el sentido de actualización permanente del Plan, brindará nuevos aportes, generando los insumos necesarios para consolidar el proceso de revisión.

A su vez los proyectos y programas identificados en el Plan requieren una ponderación según las necesidades más urgentes que, en concordancia con las asignaciones y disponibilidades presupuestarias definen el orden de prioridades, etapas y secuencias a seguir para la concreción de las obras $u$ otras acciones.

Para que esta suma de esfuerzos y aportes, esta extensa elaboración; no quede solo en la letra de una norma y su publicación, se necesita ingresar inmediatamente y avanzar en la fase de realizaciones para garantizar la utilidad del Plan como instrumento apto para dar una respuesta adecuada a las necesidades y aspiraciones de la comunidad.

\section{Bibliografía}

DE BELÁUSTEGUI, H. Encuesta Origen Destino 2009: Movilidad en el Área Metropolitana de Córdoba. Buenos Aires, Proyecto de Transporte Urbano de Buenos Aires, 2011.

GEDDES, P. Ciudades en evolución. Buenos Aires, Infinito, 1960.

IRÓS, G. Ciudad y Región: orientaciones para un desarrollo ambiental sostenible. Córdoba, Editorial FAUDI-UNC, 2007.

IRÓS, G. et al. Lineamientos para un plan estratégico urbano territorial de la región metropolitana de Córdoba. Córdoba, Ministerio de Infraestructura del Gobierno de la Provincia de Córdoba, 2012.

IRÓS, G. et al. Hacia un transporte urbano - metropolitano integrado: Propuesta para el cuadrante de Sierras Chicas, Córdoba, Argentina. Córdoba: Ministerio de Infraestructura del Gobierno de la Provincia de Córdoba, 2014.

TERZAGA, A. Geografía de Córdoba. Córdoba, Assandri, 1963.

VÁZQUEZ, J. et al. Geografía Física de la Provincia de Córdoba. Córdoba: Boldt, 1979.

Citación: IRÓS, G. et al. Plan Director para la Región de Sierras Chicas, Córdoba, Argentina. En: Libro de proceedings, CTV 2018. XII Congreso Internacional Ciudad y Territorio Virtual. "Ciudades y Territorios Inteligentes". UNCuyo, Mendoza, 5-7 septiembre 2018. Barcelona: CPSV, 2018, p. 29-44. 